\title{
A group of outbursts with exponential decays in the Rapid Burster
}

\author{
V. Šimon \\ Astronomical Institute, Academy of Sciences of the Czech Republic, 25165 Ondřejov, Czech Republic \\ e-mail: simon@asu.cas.cz
}

Received 22 January 2007 / Accepted 13 August 2008

\begin{abstract}
Aims. We present an analysis focused on a specific group of six intense outbursts (abbreviated as group A) that displays strong persistent emission in the unique low-mass X-ray binary Rapid Burster (MXB 1730-335).

Methods. Observations taken from ASM/RXTE in the 1.5-12 keV passband were utilized.

Results. We interpret the exponential decay of these outbursts as arising from irradiation of the disk by X-rays strong enough to ionize all of the disk out to its outer edge without self-shadowing, using earlier models. The parameters of irradiation apparently remained stable with a relatively high degree of consistency for all six events of group A, but they underwent a large and rapid change in twelve outbursts of the subsequent set (group B). We argue that this was because of a change in the conditions for the disk irradiation. We interpret the irradiation in terms of the slim accretion disk region. We find that the vertical dimension of the irradiating source, hence its ability to irradiate the disk, decreases with the increasing luminosity of the outbursts with exponential decays in neutron-star, soft X-ray transients of a comparable orbital period length, and is exceptionally small in the Rapid Burster. The group A outburst profiles indicate that the Rapid Burster is an ultra-compact system. The difference between the intensities and profiles of the group A and group B outbursts is not caused by a different absorption and the irradiating source remains the same for both groups. Fluctuations in the outburst profile display striking regularities, not only inside each group, but also between both groups. We interpret them as due to the spiral arms reaching the disk center. The very rapid rise of outbursts in both groups speaks in favor of a small radius for the optically thin, advection-dominated flow prior to an outside-in outburst. We argue in favor of a relatively weak magnetic field of the neutron star in this system and against a significant role for the propeller effect.
\end{abstract}

Key words. stars: neutron - accretion, accretion disks - binaries: close - circumstellar matter - X-rays: binaries stars: individual: Rapid Burster

\section{Introduction}

The Rapid Burster, MXB 1730-335, is a remarkable system (Lewin et al. 1976) lying in the globular cluster Liller 1 at the distance of $d \approx 8 \mathrm{kpc}$ (Liller 1977; Ortolani et al. 1996). This Rapid Burster is unique in displaying two types of X-ray bursts during the outbursts: type I caused by thermonuclear runaway of the accreted matter on the surface of the neutron star (NS), and type II attributed to spasmodic accretion (see e.g. Lewin et al. 1993 and Lewin et al. 1995, for reviews). It is not clear which ingredients and system parameters are needed to produce type II bursts. Among the recent models, Spruit \& Taam (1993) have proposed that the presence and profile of type II bursts depend on the mass accretion rate and the properties of the boundary layer of the magnetosphere. On the other hand, Mahasena et al. (2003) argue for a limit cycle of the accretion flow between an optically thick, advection-dominated accretion flow (ADAF) and a standard disk. Rutledge et al. (1995) show that the strength and frequencies of quasi-periodic oscillations (QPOs) in both persistent emission and bursts, and a strong anticorrelation between QPO frequency and burst peak flux distinguish the Rapid Burster from both "atoll" and "Z" sources.

The recent outbursts of the Rapid Burster reported by Guerriero et al. (1999) and Masetti (2002) appear to be similar to those in soft X-ray transients (SXTs) (see e.g. Chen et al. 1997 for a review of SXTs). The outbursts of SXTs are interpreted in terms of thermal instability of the accretion disk (e.g. van Paradijs 1996; King \& Ritter 1998; Dubus et al. 2001). Guerriero et al. (1999) analyzed four outbursts of the Rapid
Burster and show that their decaying branch can be approximated by an exponential curve, with a possible reflare. Each of these outbursts initially displayed Phase 1, i.e. a strong persistent emission with only type I bursts. This was later (after 1520 days) followed by a Phase 2 characterized by type II bursts.

In this paper we concentrate on the remarkable group of intense outbursts of the Rapid Burster and compare it to the subsequent group of less intense outbursts. We investigate their nature and the physical processes in the disk. We also compare the parameters of outbursts in the Rapid Burster with a representative group of outbursts in SXTs, and this enables us to place them into a system of outbursts in SXTs.

\section{Data source}

The recent outbursts of the Rapid Burster between 1996-2004 were observed by the All Sky Monitor $(A S M)$ onboard the Rossi X-ray Timing Explorer (RXTE) (Levine et al. 1996) (http://xte.mit.edu/). This monitor provides longterm observations of an unprecedented quality for this object. The data file contained the sum of band intensities $I_{\text {sum }}(1.5-12 \mathrm{keV})$ and the hardness ratios $H R 1=I_{\mathrm{B}}(3-5 \mathrm{keV}) / I_{\mathrm{A}}(1.5-3 \mathrm{keV})$, $H R 2=I_{\mathrm{C}}(5-12 \mathrm{keV}) / I_{\mathrm{B}}(3-5 \mathrm{keV})$.

Since our analysis concentrates on variations in the time scales of days and longer, we used the one-day means and their errors $\sigma_{\mathrm{q}}$ provided by $A S M / R X T E$. An exception is an analysis of the anomalous outburst with the maximum in JD 2451045 


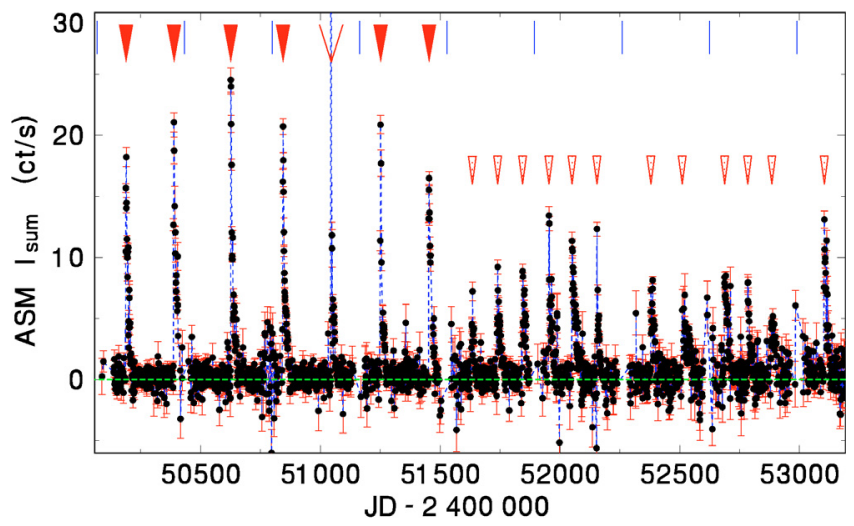

Fig. 1. X-ray light curve of the Rapid Burster as measured by the $A S M / R X T E$ sum band (1.5-12 keV) observations. Only the one-day means with $\sigma_{\mathrm{q}}$ of $I_{\text {sum }}$ smaller than $2 \mathrm{ct} \mathrm{s}^{-1}$ are plotted. They are connected by lines for the densely covered intervals. The uncertainties quoted in the original file of the ASM measurements are marked. Filled triangles denote the moments of the maxima of outbursts of group A. Open triangles after JD 2451500 mark the outbursts of group B. The outburst with the maximum in JD 2451045 belongs neither to group A or group B. The short vertical lines mark the moments of the conjunction of the system with the Sun. See Sect. 3 for details. (This figure is available in color in electronic form.)

for which also the individual $90 \mathrm{~s}$ dwells without the restriction on $\sigma_{\mathrm{q}}$ described below were used.

An inspection of the $A S M / R X T E$ lightcurve revealed that some data display a large $\sigma_{\mathrm{q}}$ and thus introduce a strong noise. It was therefore decided to set the truncation limit of $\sigma_{\mathrm{q}}$ of $I_{\text {sum }}$ at $2 \mathrm{ct} \mathrm{s}^{-1}$. This largely improved the light curves; at the same time, the coverage remained sufficiently dense. An exception is the outburst of group B with the maximum in JD 2451635, in which two data points with $\sigma_{\mathrm{q}}$ slightly exceeding $2 \mathrm{ct} \mathrm{s}^{-1}$ define the initial part of the outburst.

The truncation limit for $H R 1$ and $H R 2$ was determined in the following way. The quoted uncertainties $\sigma_{\mathrm{q}}$ of both $H R 1$ and $H R 2$ were plotted versus $I_{\text {sum }}$ and their dependence on it examined. We decided to use $H R 1$ and $H R 2$ with $\sigma_{\mathrm{q}} \leq 0.3 \mathrm{ct} \mathrm{s}^{-1}$.

\section{Data analysis}

The X-ray light curve analyzed here is shown in Fig. 1, where isolated outbursts are the dominant features. The peak intensity and the recurrence time of the outbursts, $T_{\mathrm{C}}$, decreased considerably and rapidly, in accordance with the finding by Masetti (2002). We find that this was accompanied by a large change in the profile of the X-ray curve (see also Šimon 2006). This analysis is primarily focused on a remarkable group of six intense outbursts, called group A here. When possible, only those events of this group that were observed to display Phase 1, that is those with intense persistent emission, were selected (see Guerriero et al. 1999, and their Note added in proof for details).

A way to compare the basic parameters of groups A and B is displayed in Fig. 2. It makes use of the statistical distribution of relative energy $R E$ and the peak intensity $I_{\max }$ of outbursts. The definition and meaning of $R E$ is as follows (see also Šimon 2000). When comparing the energy output of the individual outbursts in a given SXT, we can get more insight if we introduce the so-called relative energy $(R E)$ of outburst. The $R E$ of each outburst of the Rapid Burster was calculated by integration of the 1.5-12 keV X-ray lightcurve; i.e. it represents the area outlined by the outburst lightcurve in the investigated passband. The
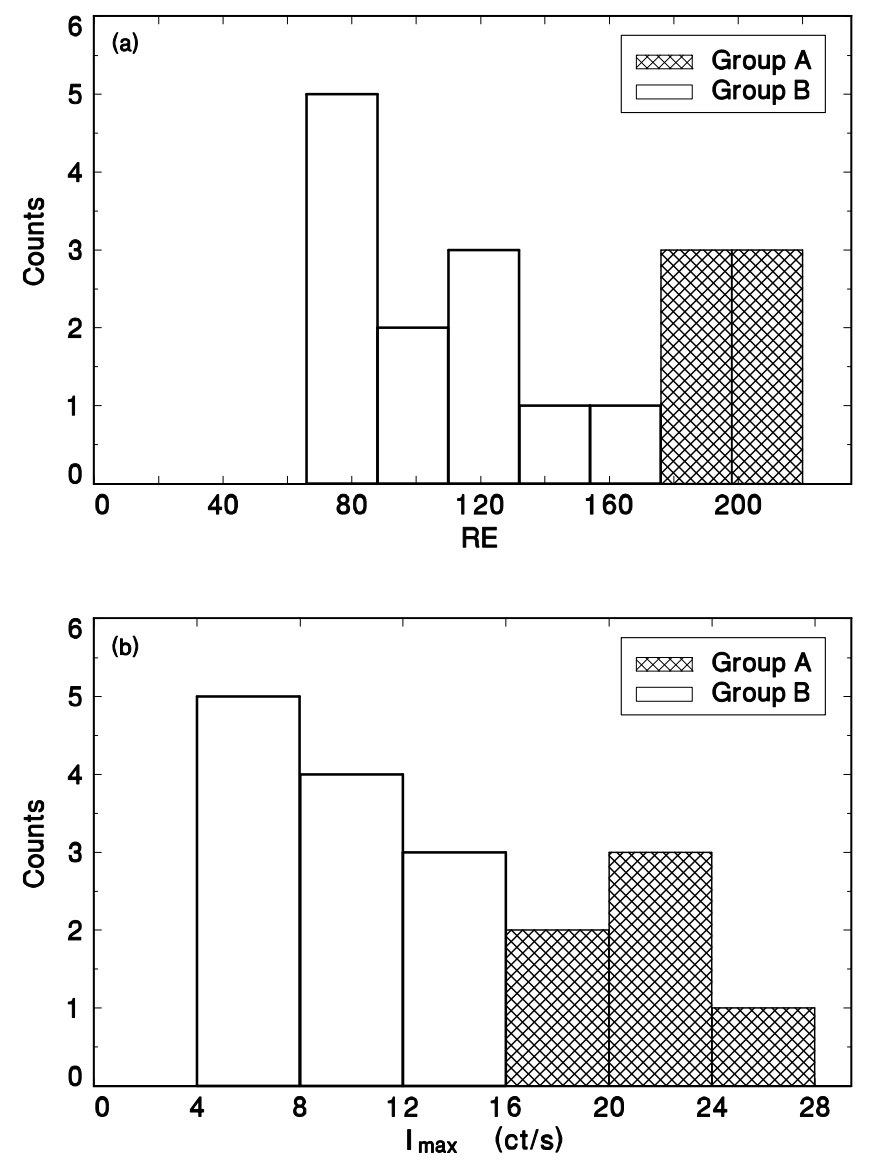

Fig. 2. The statistical distribution of $R E$ (relative energy) a) and $I_{\max } \mathbf{b}$ ) of the outbursts from Fig. 1. The groups of outbursts A and B, defined in Sect. 3, are resolved. The anomalous outburst with the maximum in JD 2451045 is not included. See Sect. 3 for details.

limits of this integration (the start and finish of the outburst) were set equal to $I_{\text {sum }}=0 \mathrm{ct} / \mathrm{s}$, so $R E$ represents the energy output of each outburst in a given energy passband. Since we are interested in comparing only the relative outputs, in a given SXT outburst, $R E$ may be expressed in dimensionless units. Our approach of calculating $R E$ is based on the assumption that persistent emission plays a significant role in both groups. Only one outburst (max. in JD 2451045 ) clearly deviates from this assumption (see below), and no $R E$ was therefore calculated for it. It can be seen that the outbursts of group A form a distinct group in Fig. 2. Both their $R E$ and $I_{\max }$ are systematically higher than those of group B. In addition, group A possesses a smaller scatter of both quantities than does group B.

A study of the morphology of outbursts can best be made by matching the events to a representative outburst, taken as a template. This procedure is similar to the method applied to the outbursts of dwarf novae (e.g. Šimon 2000) and the SXTs Aql X-1 (Šimon 2002) and 4U 1608-52 (Šimon 2004). Examinations revealed that the decaying branches of the outbursts of the Rapid Burster display fluctuations, so it was decided to modify the method. We carried out several experiments and found that the slope and profile of the rising branch remain very steep and are quite similar for the individual outbursts (Fig. 3). This branch proved to be suitable for folding the individual events.

The outburst with its maximum in JD 2450844 was chosen as the template for group A because of its smooth, well-mapped profile. The remaining outbursts were time shifted to match the rising branch of this template. The point $I_{\text {sum }}=10 \mathrm{ct} \mathrm{s}^{-1}$, on the 


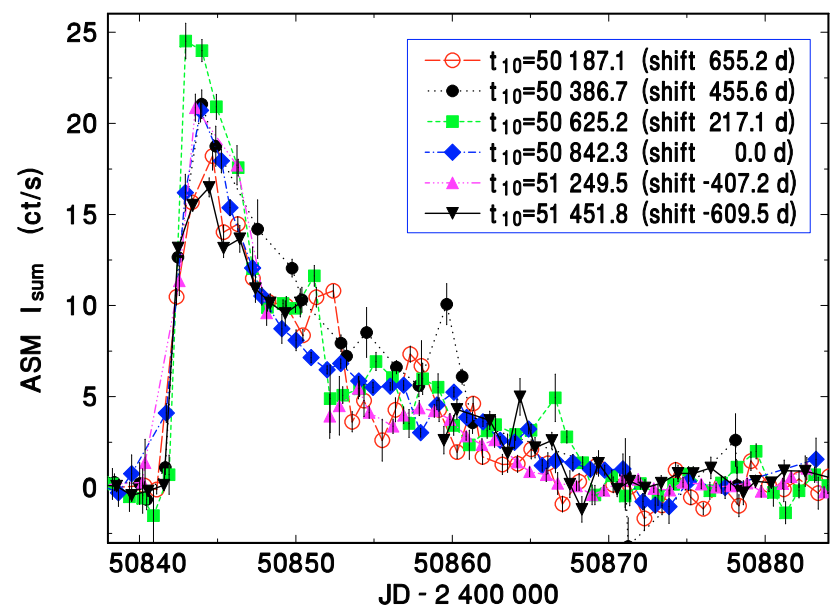

Fig. 3. A comparison of six intense outbursts of group A, marked in Fig. 1. The individual outbursts were shifted along the time axis to match the rising branch of the fourth curve template (this template has a shift of 0.0 days). The time of crossing $I_{\text {sum }}=10 \mathrm{ct} \mathrm{s}^{-1}$ on the rising branch of the outburst, $t_{10}$, given in JD-2 400000 , and the shifts (in days) with respect to the template, are listed. The points within each outburst are connected by lines for clarity. See Sect. 3 for details. (This figure is available in color in electronic form.)

rising branch of the outburst, was chosen as the reference level. The folded events are displayed in Fig. 3. It can be seen that, in spite of the fluctuations of the profile of the decaying branch of the individual outbursts, the folding reveals conspicuous similarities in the overall profile (the slope of the rising branch, the overall decay rate, the length of the outburst). The lightcurves of the individual outbursts were therefore merged into a common file and smoothed by the code HEC13, written by Prof. P. Harmanec and based on the method of Vondrák $(1969,1977)$. This code can fit a smooth curve to the data no matter what their profile. It calculates a fitted point to each observed data point, making use of two input parameters, $\epsilon$ and $\Delta T$. Here, $\epsilon$ determines how "tight" the fit will be, that is, if only the main profile or also the high-frequency variations are to be reproduced. Also, $\Delta T$ is the interval over which the data are binned before smoothing. We preferred to use this method because it does not make any presumptions about the profile of the fitted data. The X-ray curves of SXTs are often complicated (e.g. Chen et al. 1997) and a considerable part of information could be lost by fitting a particular function. We examine later in which intervals the fit approximates the curve predicted by the models.

To reproduce the sharp peak and slow decaying branch of group A without false features and large distortions, it was necessary to fit them separately and then join the fits. The fit with $\epsilon=1$ (in dimensionless units), $\Delta T=0.5$ days, was found to satisfy the rising branch and the peak of the lightcurve. On the other hand, the fit with $\epsilon=10^{-1}, \Delta T=4$ days was used for the decaying branch. Only a slight discontinuity remains, which does not influence the results. Any small rebrightening events and fluctuations are lost in this smoothing. The moving averages also enabled us to calculate the time variations of the standard deviation of the residuals of the fits, $\sigma_{\text {res }}$, and to thus obtain the measures of the variations in the lightcurve in various phases of the outburst. The values of $\sigma_{\text {res }}$ were calculated from the residuals of the HEC13 curves using the two-sided moving averages with the filter half-width $Q=4 \mathrm{~d}, 5 \mathrm{~d}, 6 \mathrm{~d}$ in steps of 0.1 day (Fig. 4c).
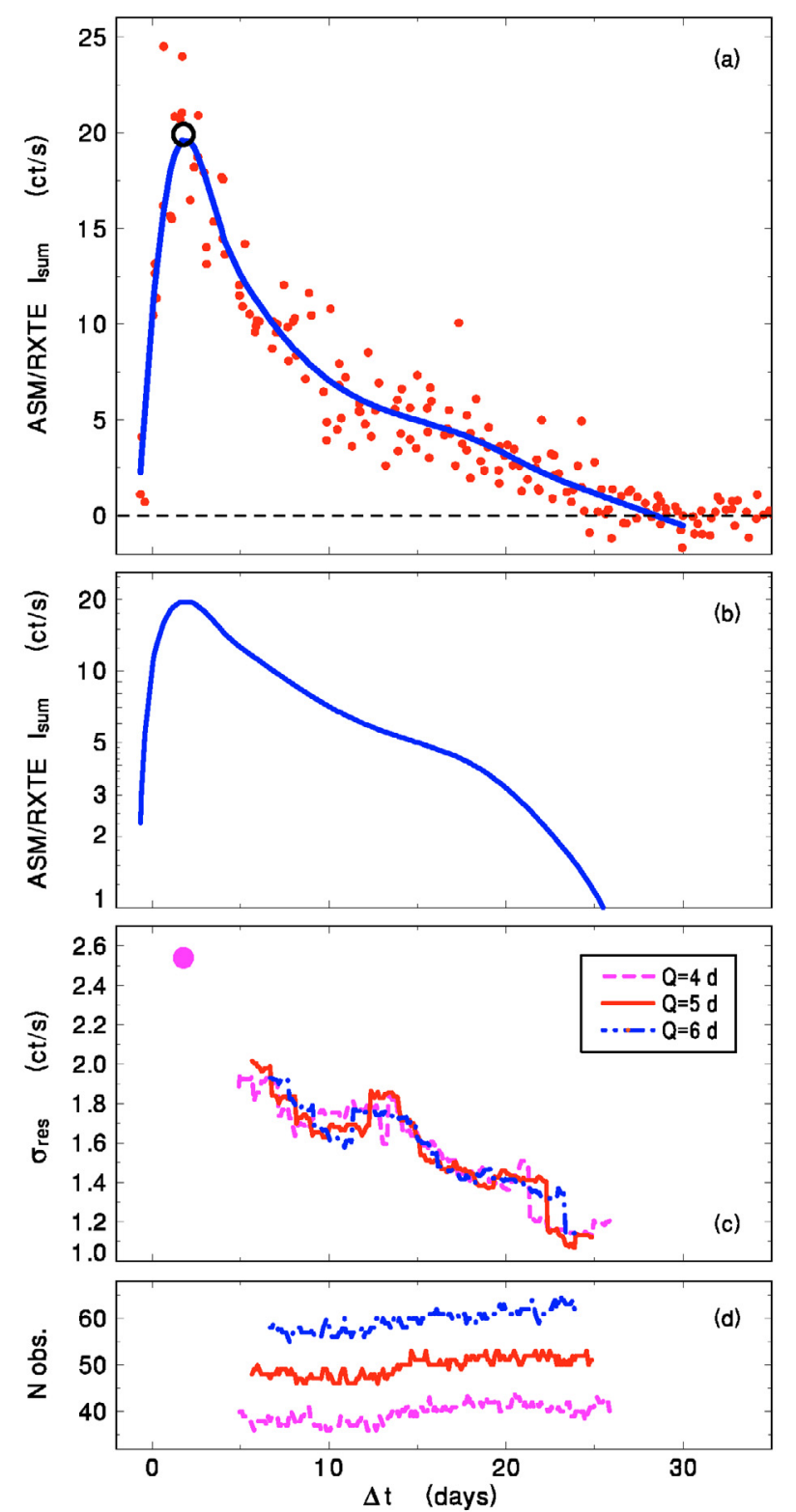

Fig. 4. a) Merged $A S M / R X T E$ sum band light curves of the outbursts of group A from Fig. 3. $\Delta t$ gives the time interval with respect to crossing $I_{\text {sum }}=10 \mathrm{ct} \mathrm{s}^{-1}$ on the rising branch. The folded light curves were smoothed by the code HEC13 (smooth curve). The large open circle represents the mean $I_{\text {sum }}$ of the averaged maxima of six outbursts. b) The fit to the merged light curve (logarithmic scale). c) The residuals $\sigma_{\text {res }}$ of the fit smoothed by two-sided moving averages for $Q=4 \mathrm{~d}, 5 \mathrm{~d}$, $6 \mathrm{~d}$. The large solid circle represents the standard deviation of the mean of the averaged maxima of six outbursts. d) The number of observations included in the calculation of each mean. See Sect. 3 for details. (This figure is available in color in electronic form.)

The mean $I_{\text {sum }}$ of the maxima of six outbursts of group A and its standard deviation, not the mean of the individual measurements, is included in Figs. 4a and c. This approach avoids false features that appear near the edge of the data set and removes the artificially large $\sigma_{\text {res }}$ in this phase, caused by the moments of the maxima of the individual outbursts not occurring exactly at the same time with respect to $t_{10}$.

Difficulties arise in classifying the profiles of the decaying branches of those outbursts that occurred after group A (abbreviated here as group B), partly because of fluctuations in $I_{\text {sum }}$ 


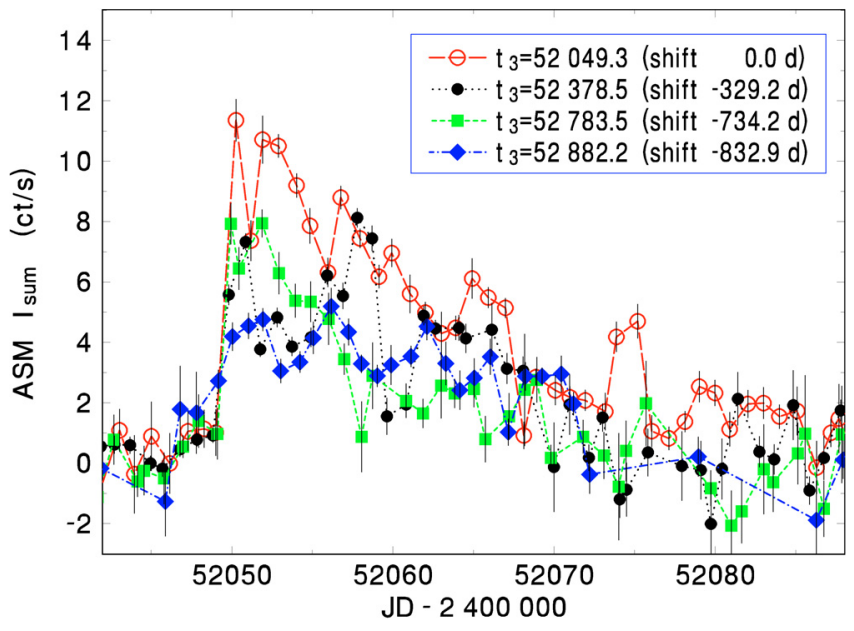

Fig. 5. Examples of the outbursts of group B, marked in Fig. 1. The arrangement is analogous to Fig. 3 , but the crossing of $I_{\text {sum }}=3 \mathrm{ct} \mathrm{s}^{-1}$ on the rising branch was used as the reference point. See Sect. 3 for details. (This figure is available in color in electronic form.)

on timescales of days. We use group B to show the large difference in the decay profile of outbursts that followed group A. It is not clear if the outbursts of group B always displayed Phase 1 like group A. Only one outburst of group B was observed by Falanga et al. (2004) to search for fast variability characteristics, but types I and II could not be conclusively distinguished. However, the ASM/RXTE curves of group $\mathrm{B}$, consisting of daily means, display a steep rise to $I_{\max }$, on average followed by a slow decline with fluctuations superposed. That lightcurves of the one-day means of $I_{\text {sum }}$ for group B outbursts are not fully chaotic indicates that the $1.5-12 \mathrm{keV}$ emission is caused to a significant extent by the persistent emission in a similar way to group A. Folding like for the group A outbursts was also carried out on the outbursts of group B (Fig. 5). Only 4 of 12 outbursts are plotted to avoid overcrowding of the plot. Since these events are fainter than those of group A by a factor of two to four, one has to be careful, because the rising branch is sometimes so steep that it is not covered by the one-day mean. However, it was found that this uncertainty in the onset time is considerably less than the duration of the outburst itself. The lightcurves of group B were subject to the same smoothing by the code HEC13 as in group A. The fit with $\epsilon=10, \Delta T=0.5$ days was found to satisfy the rising branch and the peak of the lightcurve, while the fit with $\epsilon=10^{-1}, \Delta T=7$ days was used for the decaying branch (Fig. 6a).

The profiles of $\sigma_{\text {res }}$ are in good mutual agreement for all three values of $Q$ in each group (Figs. $4 \mathrm{c}$ and $6 \mathrm{~b}$ ), although the mean profiles of the X-ray lightcurves are largely discordant (Figs. 4a and 6a). Generally, $\sigma_{\text {res }}$ decreases as the flux decreases, with a possible plateau or a change in slope 10-13 days after the peak. A comparison of Fig. 4 with the position of the possible reflares proposed by Guerriero et al. (1999) shows that the coincidence of such reflares with the plateau and its surrounding steps can at most be marginal.

The relation between $I_{\text {sum }}$ and $\sigma_{\text {res }}$ from Figs. 4 and 6 during the outburst decline is displayed in Fig. 7. Notice that $\sigma_{\text {res }}$ attains a similar value at a given $I_{\text {sum }}$ for both groups. The point that denotes $I_{\text {sum }}$ of the peak intensity of the individual group A outbursts indicates that the decay in $\sigma_{\text {res }}$ is faster in the early phase and slows down later on.

An inspection of Figs. 3 and $4 \mathrm{a}$ shows that the rising branches of outbursts in the Rapid Burster are very steep. The

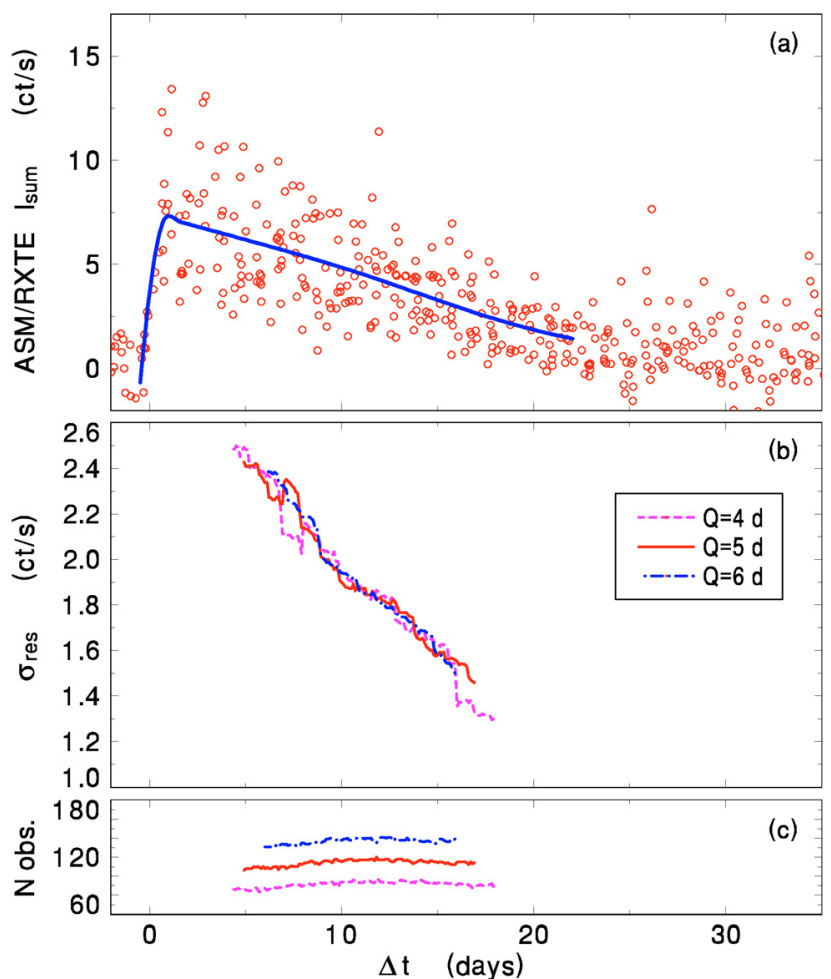

Fig. 6. Merged and smoothed ASM/RXTE sum band lightcurves of 12 outbursts of group B. The arrangement is analogous to Fig. 4. $\Delta t$ gives the time interval with respect to crossing $I_{\text {sum }}=3 \mathrm{ct} \mathrm{s}^{-1}$ on the rising branch. See Sect. 3 for details. (This figure is available in color in electronic form.)

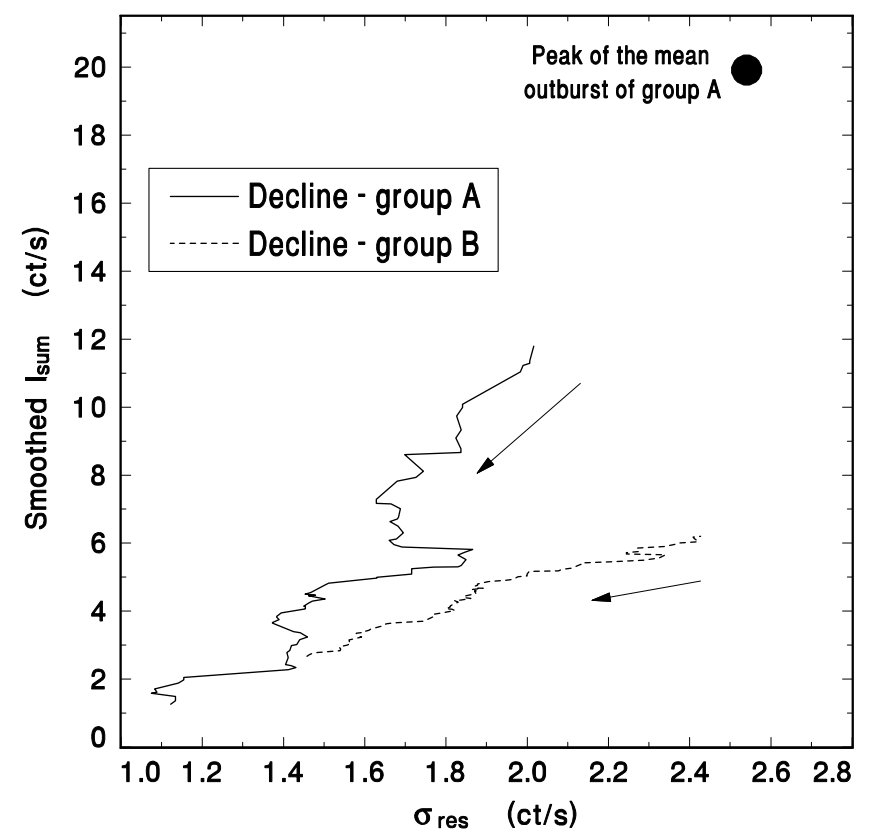

Fig. 7. The relation between the smoothed $I_{\text {sum }}$ and $\sigma_{\text {res }}$ in the outbursts of groups A and B during the decline of the flux from the outburst maximum. The smoothing for $Q=5 \mathrm{~d}$ was used. The arrows denote the time evolution for convenience. See Sect. 3 for details.

slope of this branch was studied using a comparison with those of the outbursts of the NS SXTs Aql X-1 and 4U 1608-52, observed by $A S M / R X T E$, which is in the same spectral passband. Several outbursts were merged for each SXT: the data 


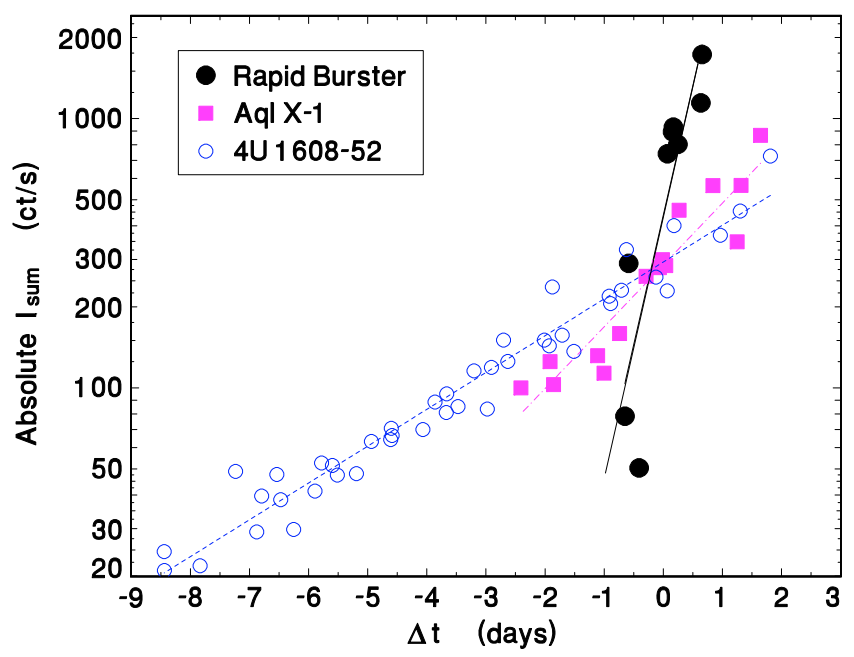

Fig. 8. The rising branches of the outbursts of the NS SXTs, observed by $A S M / R X T E$ in the $1.5-12 \mathrm{keV}$ passband. The count-rates were recalculated for the distance of the object and corrected for interstellar extinction. Several outbursts were merged for each SXT. A logarithmic scale was used for the ordinate. See Sect. 3 for details. (This figure is available in color in electronic form.)

from Šimon (2002) and Šimon (2004) were used for Aql X-1 and 4U 1608-52, respectively. To make a reasonable comparison of these SXTs, the count-rates were re-calculated for the distance of the object and corrected for the interstellar extinction as follows - the Rapid Burster: $d=8 \mathrm{kpc}$ (Ortolani et al. 1996), $N_{\mathrm{H}}=1.5 \times 10^{22} \mathrm{~cm}^{-2}$ (Guerriero et al. 1999); Aql X-1: $d=4 \mathrm{kpc}$ (Rutledge et al. 2001), $N_{\mathrm{H}}=3.4 \times 10^{21} \mathrm{~cm}^{-2}$ (Maitra \& Bailyn 2004); 4U 1608-52: $d=3.6 \mathrm{kpc}$ (Nakamura et al. 1989), $N_{\mathrm{H}}=1.5 \times 10^{22} \mathrm{~cm}^{-2}$ (Gierlinski \& Done 2002). The resulting plot is shown in Fig. 8.

As noted above, the outbursts of group B are fainter than those of group A by a factor of two to four, hence it is more difficult to analyze their rising branches. Sometimes this branch is not covered by the one-day mean at all. Nevertheless, it can be concluded that the very steep rises are also present in group B.

The outburst with the JD 2451045 maximum, surrounded by group A, was not included in the analysis of either group A or group B. The reason is that, according to Guerriero et al. (1999), it displayed only Phase 2. Indeed, our analysis revealed that this outburst possesses an anomalously bright, narrow peak and a profile divergent from the other events. The profile of this anomalous outburst is displayed in Fig. 9. A comparison with the mean profile of the group A outbursts shows that the greatest difference in the lightcurves occurs in the first half of the outburst.

To make a quantitative analysis and to also facilitate a comparison with outbursts prior to the $A S M / R X T E$ era and those in other systems, we convert the X-ray flux of the Rapid Burster in the following way according to Guerriero et al. (1999):

$3 \times 10^{-12} \mathrm{erg} \mathrm{cm}^{-2} \mathrm{~s}^{-1}$ per 1 PCA ct s${ }^{-1}(2-20 \mathrm{keV})$.

The intensity of Crab is $I_{\mathrm{CrabPCA}} \approx 13000 \mathrm{ct} \mathrm{s}^{-1}$ for $P C A / R X T E$ $(2-60 \mathrm{keV})$ (Shirey et al. 1996). However, the bulk emission lies in the $2-20 \mathrm{keV}$ passband. At the same time, $I_{\text {CrabASM }}=75 \mathrm{ct} \mathrm{s}^{-1}$ for $A S M / R X T E$. The intensity of the Rapid Burster at the peak of outburst of group A, determined from the fitted profile in Fig. 4 , is then $I_{\max }=19 \mathrm{ct} \mathrm{s}^{-1}=0.253 \mathrm{Crab}=3290 \mathrm{PCA}$ ct s${ }^{-1}$, which yields the peak flux $F_{\text {Mobs }}=9.9 \times 10^{-9} \mathrm{erg} \mathrm{cm}^{-2} \mathrm{~s}^{-1}$. Correction for the extinction $N_{\mathrm{H}}=1 \times 10^{22} \mathrm{~cm}^{-2}$ using the spectra of the persistent emission by Masetti et al. (2000) and

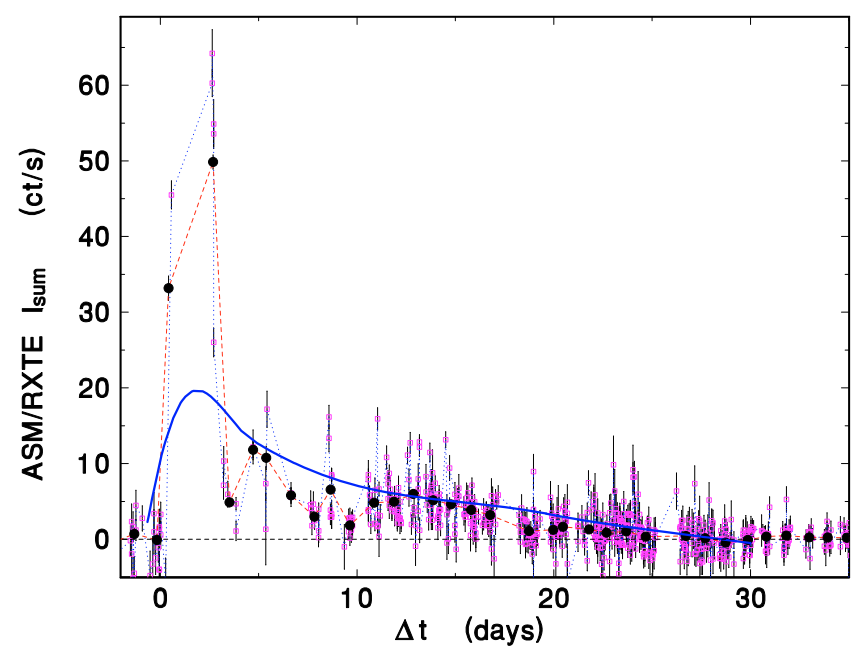

Fig. 9. The profile of the anomalous outburst with the maximum in JD 2451045 and $t_{10}$ in JD 2451 043.5. The solid circles mark oneday means (the error bars of some measurements being smaller than the size of the symbol), while open boxes denote the individual dwells. The individual points are connected by lines for clarity. The mean profile of outbursts from Fig. 4a is shown for comparison. Notice the difference mainly in the first half of the outburst profile. See Sect. 3 for details. (This figure is available in color in electronic form.)

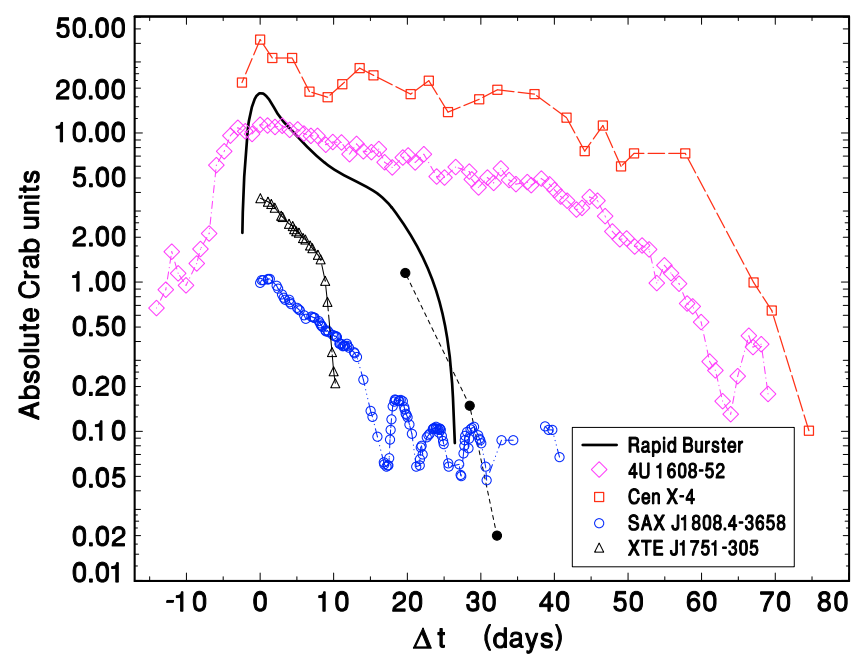

Fig. 10. Outbursts with exponential decaying branches in NS SXTs. The smooth curve represents the fit to the mean profile of group A outbursts in the Rapid Burster, while closed circles denote the final decline of the 1998 February-March outburst observed by Masetti et al. (2000). $\Delta t$ denotes the time interval with respect to the moment of the maximum $I_{\mathrm{ACU}}$ or to the start of observation. All the intensities were transformed into absolute Crab units. A logarithmic scale was used for the ordinate. See Sect. 3 and Table 1 for details. (This figure is available in color in electronic form.)

the cross sections by Morrison \& McCammon (1983) increases the flux in the $0.7-12 \mathrm{keV}$ passband by a factor of only $\sim 1.25$ with respect to the flux observed in the $1.5-12 \mathrm{keV}$ passband (provided that the spectrum of the persistent emission remains roughly similar throughout the outburst). The luminosity at the outburst peak is then $L_{\max } \approx 9.5 \times 10^{37} \mathrm{erg} \mathrm{s}^{-1}$.

Outbursts with prominent exponential decaying branches are rare in NS SXTs. Five such systems are displayed in Fig. 10 and help us put the group A outbursts in the Rapid Burster into context. For the sake of consistency, all the intensities were 
Table 1. Parameters of NS SXTs and their outbursts with exponential decaying branches, displayed in Fig. 10. See text for details.

\begin{tabular}{lrcrcrrl}
\hline \hline System & $\begin{array}{r}P_{\text {orb }} \\
(\mathrm{hr})\end{array}$ & $\begin{array}{c}d \\
(\mathrm{kpc})\end{array}$ & $\begin{array}{r}\text { Source } \\
\text { of LC }\end{array}$ & Max. $I_{\mathrm{ACU}}$ & $I_{\mathrm{T}}$ & $\begin{array}{r}\tau_{\mathrm{d}} \\
(\mathrm{d})\end{array}$ & $\begin{array}{l}E \text { range } \\
(\mathrm{keV})\end{array}$ \\
\hline Rapid Burster & & $8.0[1]$ & & 18.4 & $\sim 3.0$ & 8.2 & $1.5-12$ \\
4U 1608 - 52 & $12.89[2]$ & $3.6[3]$ & {$[4]$} & 11.4 & 4.7 & 40 & $1.5-12$ \\
Cen X-4 & $15.1[5]$ & $1.2[6]$ & {$[7]$} & 42 & 7.2 & 38 & $3-12[7]$ \\
SAX J1808.4-3658 & $2.014167[8]$ & $3.5[9]$ & {$[10]$} & 1.05 & 0.33 & 11.3 & $2-16[10]$ \\
XTE J1751-305 & $0.71[11]$ & $(8.5)[12]$ & {$[10]$} & 3.66 & 1.50 & 8.6 & $2-16[10]$ \\
\hline
\end{tabular}

References: 1. Ortolani et al. (1996); 2. Wachter et al. (2002); 3. Nakamura et al. (1989); 4. Šimon (2004); 5. Chevalier et al. (1989); 6. Kaluzienski et al. (1980); 7. Chen et al. (1997); 8. Chakrabarty \& Morgan (1998); 9. Galloway \& Cumming (2006); 10. Wijnands (2006); 11. Markwardt et al. (2002); 12. Gierlinski \& Poutanen (2005).

transformed into absolute Crab units, $I_{\mathrm{ACU}}$. Equation (1) was used for the Rapid Burster, 4U 1608-52, and Cen X-4:

$I_{\mathrm{ACU}}=\frac{I_{\mathrm{sum}}}{I_{\mathrm{CrabASM}}} d^{2} \frac{1}{A}$.

Equation (2) was used for SAX J1808.4-3658 and XTE J1751305:

$I_{\mathrm{ACU}}=I_{\mathrm{C}} d^{2}$,

where $I_{C}$ is expressed in Crabs, as used in the original references in Table 1 , distance $d$ is expressed in kpc, and $A$ denotes what fraction of the flux remains after taking the interstellar absorption according to Morrison \& McCammon (1983) into account. The decrease in intensity was found to be quite small $(<10$ percent) for the objects considered and their spectral ranges. It therefore does not influence our results.

Parameters of NS SXTs and their outbursts with exponential decaying branches are summarized in Table 1 . The LC denotes the X-ray lightcurve, $I_{\mathrm{T}}$ refers to the intensity of the transition of the decaying branch from an exponential to a linear profile (expressed in ACU), $\tau_{\mathrm{d}}$ is the decay time scale ( $e$-folding time) of the outburst. The numbers in the squared brackets denote the references. If no number is given, the relevant value was determined by our analysis.

Both $H R 1$ and $H R 2$ of both groups are plotted versus $I_{\text {sum }}$ in Fig. 11, and $H R$ of group A were fitted by HEC13 as a function of $I_{\text {sum. }}$. To avoid false features in the fitting curve, two data points with the highest $I_{\text {sum }}$ were omitted before fitting. The value $\epsilon=1$ was used for both groups, and $\Delta I_{\text {sum }}=5.3$ and 5.5 were found to provide satisfactory fits for groups $\mathrm{A}$ and $\mathrm{B}$, respectively. In spite of a scatter, a well-defined trend toward hardening of the group A $H R 1$ with the intensity can be resolved for $I_{\text {sum }}<12 \mathrm{ct} \mathrm{s}^{-1}$. On the other hand, the fit shows no change in

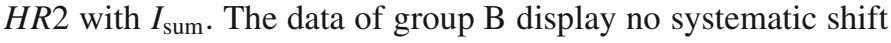
in $H R$ with respect to those of group A.

Plotting the maximum luminosity $L_{\max }$ vs. $L_{\mathrm{q}} / L_{\max }$ of outburst of SXTs ( $L_{\mathrm{q}}$ being the quiescent luminosity) can help us assess if and how the properties of the Rapid Burster (e.g. the role of the propeller effect) differ from those of other SXTs. This diagram, displayed in Fig. 12, is based on those by Narayan et al. (1997), Garcia et al. (1998), and Robinson et al. (2002). The position of Aql X-1 was re-calculated for $d=4 \mathrm{kpc}$ (Rutledge et al. 2001). The luminosities of additional systems come from these authors: SAX J1808.4-3658 (in't Zand et al. 2001; Campana et al. 2002), 1H 1905+000 (Jonker et al. 2006), XTE J1807294 (Campana et al. 2005, 2003), XTE J1709-267 (Jonker et al. 2003). The value of $L_{\mathrm{q}}$ of the Rapid Burster observed by Asai et al. (1996) turns out to be $2.4 \times 10^{33} \mathrm{erg} \mathrm{s}^{-1}$, when re-calculated for the $0.7-12 \mathrm{keV}$ passband with the details of the conversion
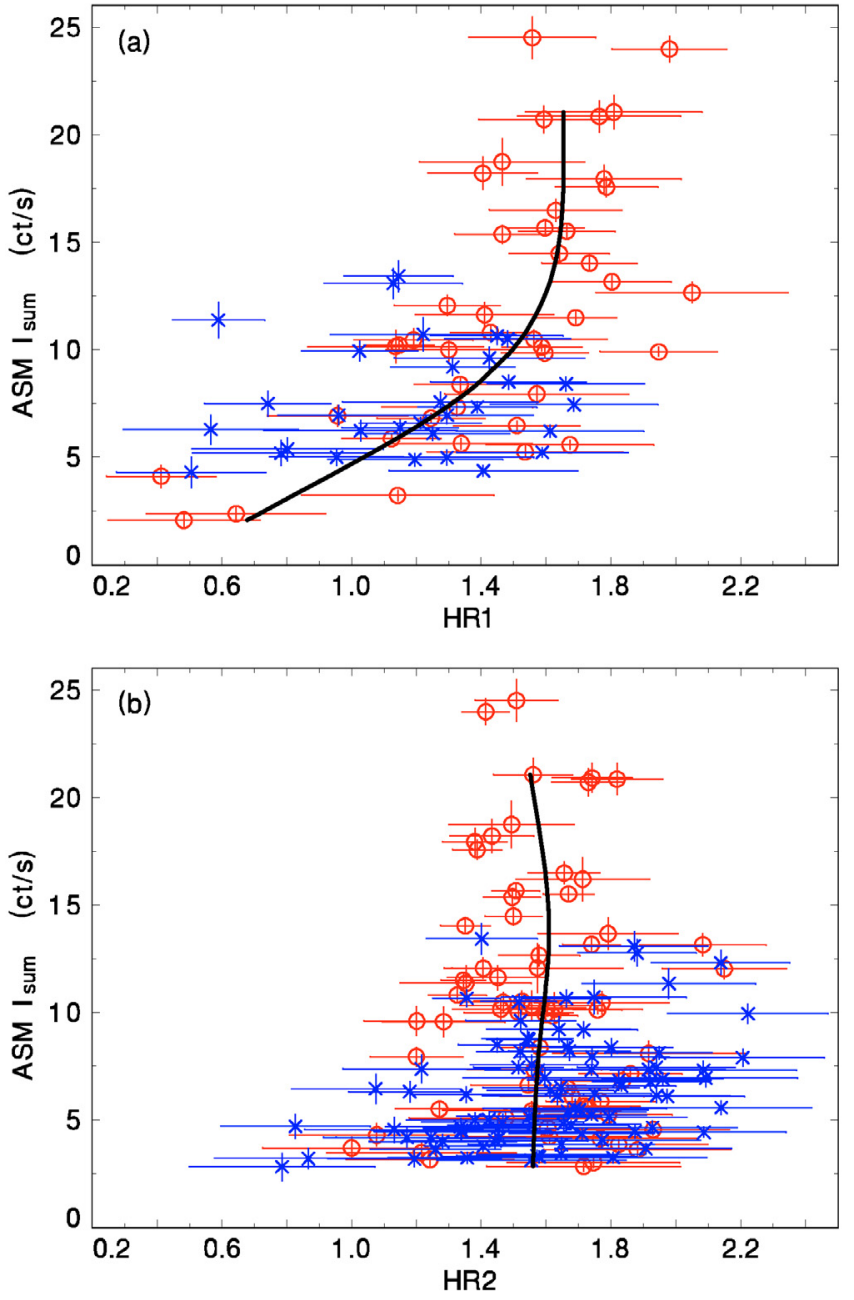

Fig. 11. The dependence of $H R 1$ and $H R 2$ on $I_{\text {sum }}$ in the Rapid Burster. Open circles and crosses refer to groups $\mathrm{A}$ and $\mathrm{B}$, respectively. The scales of the axes are identical for both diagrams. The smooth curves represent the HEC13 fits for group A. See Sect. 3 for details. (This figure is available in color in electronic form.)

given above. The position of the Rapid Burster determined for the fit to the outbursts of group A, group B, and the one for the peak of the anomalous outburst are displayed in Fig. 12. The position of another system that displays type II bursts, GRO J174428 (e.g. Kouveliotou et al. 1996), is also displayed for comparison. Its bolometric $L_{\max }=9 \times 10^{38} \mathrm{erg} \mathrm{s}^{-1}$, assuming $d=8 \mathrm{kpc}$ (Giles et al. 1996), while $L_{\mathrm{q}} \approx 3 \times 10^{33} \mathrm{erg} \mathrm{s}^{-1}$ in the $0.5-10 \mathrm{keV}$ passband (Wijnands \& Wang 2002). 


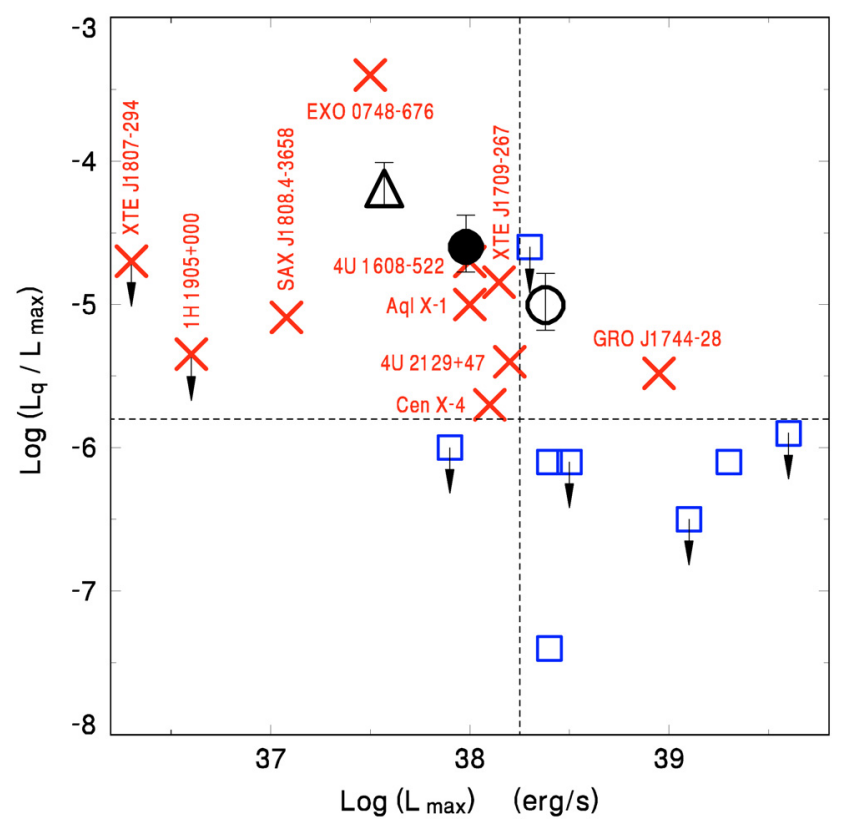

Fig. 12. The maximum luminosity $L_{\max }$ vs. ratio of the quiescent and maximum luminosity of SXTs. The NS and black hole $(\mathrm{BH})$ accretors are marked by crosses and open boxes, respectively. The names of the NS SXTs are given for convenience. A large solid circle denotes the position of the Rapid Burster determined by the fit to the group A outbursts. The open circle marks the position of this system for the peak of the anomalous outburst in Fig. 9. The open triangle denotes the location of the Rapid Burster for the group B outbursts. See Sect. 3 for details. (This figure is available in color in electronic form.)

\section{Discussion}

We present an analysis of the Rapid Burster in the state of activity in which intense persistent emission dominates during outburst.

We classify the group A outbursts as type FRED (fast rise, exponential decay) in the notation of outbursts of SXTs by Chen et al. (1997). There is an increasing line of evidence that a limiting luminosity of the persistent emission $L \approx 2 \times 10^{37} \mathrm{erg} \mathrm{s}^{-1}$ (from $I_{\text {sum }} \approx 4 \mathrm{ct} \mathrm{s}^{-1}$ ), above which type II bursts do not occur, exists in the outbursts of the Rapid Burster (Guerriero et al. 1999; Barr et al. 1987). Both the groups A and B outbursts fulfill this criterion. We interpret the properties of the group A outbursts as caused by a mass accretion rate onto the NS giving rise to a persistent emission that exceeds the limiting value above which irradiation of the disk is enabled. The group A outburst emission is fainter than the peak luminosity of type II bursts in every phase of the 1979 outburst observed by Kunieda et al. (1984). The absence of type II bursts in group A cannot be explained by outshining these bursts by persistent emission because its luminosity is lower than that of type II bursts. We interpret the extraordinary, narrow maximum of the anomalous outburst in JD 2451045 (Fig. 9) as a type II burst, and not the persistent emission.

To classify the group A outbursts, we compared their parameters with those of the ensemble of SXTs studied by Chen et al. (1997), where, $L_{\max }$ of group A is typical of the outbursts of this ensemble of SXTs with both the NS and BH accretors. When only the brightest event in a given SXT is considered, $L_{\max }$ of group A ranks in the top third of the most luminous NS SXTs on a logarithmic scale. The $L_{\mathrm{max}} / L_{\mathrm{q}}$ ratio of about $4 \times 10^{4}$ is also typical of SXTs with NS accretors. The rise time scale $(e$-folding

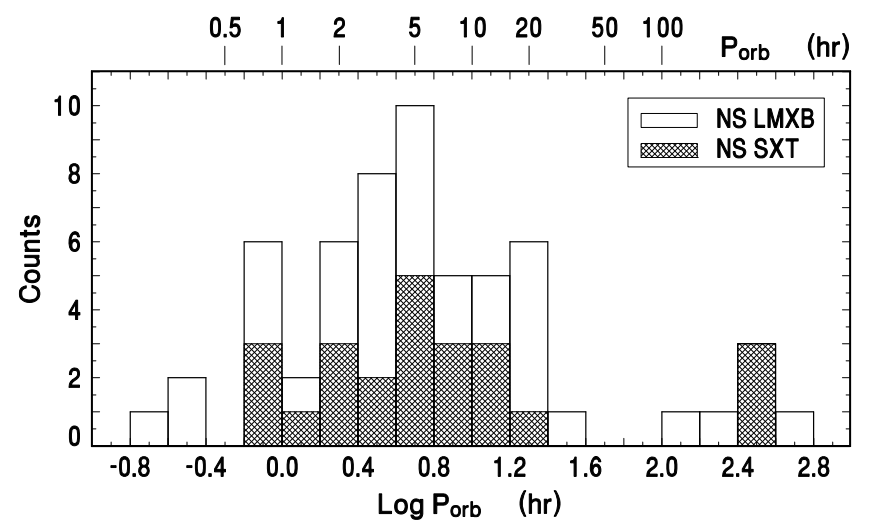

Fig. 13. The statistical distribution of $P_{\text {orb }}$ of NS LMXBs. Data from the catalog by Ritter \& Kolb (2003) and its online updates were used. See Sect. 4 for details.

time), $\tau_{\mathrm{r}} \approx 0.44 \mathrm{~d}$, and $\tau_{\mathrm{d}}=8.0 \mathrm{~d}$ place the outbursts of the Rapid Burster among SXTs with the fastest outbursts. The total energy radiated during outburst of group $\mathrm{A}$ is $E_{\mathrm{tot}} \approx 7 \times 10^{43} \mathrm{erg}$. They thus fall into about the middle of the range of SXT outbursts on a logarithmic scale of $E_{\text {tot }}$, when both NS and $\mathrm{BH}$ SXTs are considered. The group A outburst parameters thus fall into the ensemble of outbursts in SXTs with various orbital periods $P_{\text {orb }}$, and hence various disk sizes. The donor star, and the conditions inside it, play a large role in this outbursting activity of the Rapid Burster, as of SXTs in general, irrespective of the disk size. The conditions in the donor are influenced by the processes operating on a much shorter time scale than the evolutionary one. This emerges from the finding that the occurrence of the thermal instability does not depend on $P_{\text {orb }}$ in NS LMXBs (Fig. 13). A large scatter of the mass transfer rate $\dot{m}_{\text {tr }}$ at a given $P_{\text {orb }}$ thus must be common in NS LMXBs.

A very steep rising branch is characteristic of the outbursts of group A and group B. Even the crude profile of the 1979 outburst (Kunieda et al. 1984) suggests that this also occurred prior to the onset of group A. This kind of rise thus appears to be a general feature of outbursts in the Rapid Burster. A comparison with other well-observed NS SXTs, Aq1 X-1 and 4U 1608-52 (Fig. 8), shows that each of them preserves its own $\tau_{\mathrm{r}}$ for the individual outbursts, and $\tau_{\mathrm{r}}$ does not depend on the luminosity of the outburst in a given system (Šimon 2002, 2004). The steep rise to the outburst maximum can be explained by an evaporated inner disk region prior to the outburst in the framework of the model by Dubus et al. (2001). A smaller radius of the optically thin ADAF immediately prior to the outburst yields a more rapid rise. Since no second, slower phase of the rise to the outburst peak is seen in the Rapid Burster, its disk is smaller than in Aql $\mathrm{X}-1$ and $4 \mathrm{U} 1608-52$ and/or the outburst is outside-in. Since it is reasonable to assume that the mass of the accretor is close to $1.4 M_{\odot}$ in Fig. 8, each of these SXTs possesses a specific size of optically thin ADAF that is reproduced in the individual quiescent intervals.

We interpret the exponential decay of group A outbursts as arising from the irradiation of the disk by X-rays strong enough to ionize the entire disk, using the model by King \& Ritter (1998) and Dubus et al. (2001). These two approaches give similar outburst profiles in spite of the different assumed efficiencies (Truss $\&$ Done 2006), and their outburst profile is confirmed by the 2D smoothed particle model by Truss et al. (2002). The geometry plays a large role in irradiation. The unirradiated disk is supposed to be geometrically thin and concave, while the model for 
the irradiated disk by Dubus et al. (1999) predicts a puffed-up, convex disk that is self-shielded from the X-rays if they come from a point-like source.

The profiles of the group A outbursts show that there is no self-shadowing of the disk at maximum, using the 2D model by Truss et al. (2002). Either the irradiating source is thus vertically extended or the outer disk region is really concave. The relatively strong mutual similarity of the decaying branches of these outbursts suggests that both the profile of the disk and the properties of the irradiating source are reproduced with a high degree of consistency. The small rebrightenings on the decaying branch of some outbursts can be associated with a small degree of selfshadowing near the disk's outer edge in later phase of outburst.

Putting the profiles of the group A outbursts into the context of the outbursts in NS SXTs that display exponentially decaying branches sheds more light on the process of irradiation. Figure 10 and Table 1 observationally confirm the statement of the model by King \& Ritter (1998) that $\tau_{\mathrm{d}}$ depends on $P_{\text {orb }}$ in the sense that the outbursts of SXTs with longer $P_{\text {orb }}$ decay more slowly. For systems with similar $P_{\text {orb }}$, the exponentially decaying branch has a similar decay time, while the peak luminosities, hence subsequent luminosities for a given $\Delta t$, differ; the outburst of the ultra-compact system SAX J1808.4-3658 is considerably less luminous than that of another ultra-compact XTE J1751-305 (unless $d$ of XTE J1751-305 is largely overestimated). Large systematic differences in the outburst luminosities between SXTs with long, mutually similar $P_{\text {orb }}$ also occurred for $4 \mathrm{U} 1608-52$ and Cen X-4. This implies large differences in the irradiating sources. The $\tau_{\mathrm{d}}$ of the group A outbursts is very similar to that in the ultra-compact systems SAX J1808.4-3658 and XTE J1751-305, which indicates that the Rapid Burster is an ultra-compact system, too. The vertical dimension of the irradiating source decreases with the increasing luminosity, since the intensity of the transition of the decaying branch from an exponential to a linear profile, $I_{\mathrm{T}}$, differs even for the systems with similar $P_{\text {orb }}$ (Fig. 10). The change of the profile in $I_{\mathrm{T}}$ occurs at a higher luminosity for the outbursts with higher $L_{\max }$. This is definitely true for SAX J1808.4-3658 and XTE J1751-305 and appears to also occur for the Rapid Burster. We propose that a corona, manifesting itself by a hard power-law component in the X-ray spectrum (e.g. Gogus et al. 2007), which could act as an extended source irradiating the disk, is replaced by the slim accretion disk (modeled by Abramowicz et al. 1988) at high luminosities. This is consistent with the fact that persistent X-ray emission of the Rapid Burster is dominated by two blackbodies, with at most a small power-law contribution (e.g. Guerriero et al. 1999; Masetti et al. 2000). Since the luminosity of the Rapid Burster is high among NS SXTs (see above) and is near the Eddington limit modified for the accretion disk (Heinzeller \& Duschl 2007) in these outbursts, a slim optically thick inner disk region (Abramowicz et al. 1988; proposed by Mahasena et al. 2003, for type II bursts) can form, serve as the source of the persistent emission and irradiate the outer disk region in the group A outbursts.

Group A finished abruptly, as emerges from several findings. Both groups (A and B) overlap in neither $R E$ nor $I_{\max }$ (Fig. 2). Also the mean profile of the subsequent group $B$ is remarkably different from that of group A. This is also true of the individual group B outbursts. We interpret this as due to a change in the conditions for the disk irradiation. The luminosity of group B gets close to the limit when the irradiation is too weak to ionize the entire disk. The outbursts of two ultra-compact SXTs in Fig. 10 still display the exponential decay well below $I_{\mathrm{T}}$ of group $\mathrm{A}$, hence their disks are still ionized out to their outer rim. This is especially true for SAX J1808.4-3658. This is direct evidence of the exceptionally small irradiation in the Rapid Burster. If the irradiation of the disk in SAX J1808.4-3658 and XTE J1751-305 were as low as in the Rapid Burster, the decaying branches of their outbursts would no longer be exponential. The models agree on a linear decay that is considerably steeper than an exponential one in the case of a weak irradiation unable to keep the whole disk ionized. This could explain the decays in the individual fluctuations in the group B outbursts. But one has to be careful here since there is no available model to treat the disk behavior in the case of a repeating weak irradiation. Alternatively, the re-flares of non-irradiated disk, which are caused by the surface density behind the cooling front being high enough to develop a new heating front, as predicted by the model by Dubus et al. (2001), could reproduce the basic profile of the fluctuations. In our interpretation, the decreasing irradiation of the disk in the Rapid Burster gives rise to the sequence of profiles of the group A outbursts with exponential decay, the group B events with large fluctuations, and the outbursts with the dominant type II bursts and absent or weak disk irradiation.

The mean spectral parameters of both group A and group B outbursts remain stable when $I_{\text {sum }}>12 \mathrm{ct} \mathrm{s}^{-1}$, i.e. $L_{1.5-12 \mathrm{keV}}>$ $5 \times 10^{37} \mathrm{erg} \mathrm{s}^{-1}$ (Fig. 11). This implies a very stable mean X-ray spectrum in the $3-12 \mathrm{keV}$ passband, with the profile almost identical for both groups. Going below $L_{1.5-12 \mathrm{keV}} \approx 5 \times 10^{37} \mathrm{erg} \mathrm{s}^{-1}$, another component becomes increasingly more prominent in the $1.5-3 \mathrm{keV}$ passband in both groups. This implies that the difference between the intensities and profiles of the group A and group B outbursts is not caused by a different absorption and that the irradiating source remains the same for both groups.

Fluctuations in the outburst profile display striking regularities not only inside each group, but also between both groups. The $\sigma_{\text {res }}$ curve of the decaying branches of outbursts of both groups in the Rapid Burster evolves with the progress of outburst in a similar way. Both the rapid variability on the time scale of a few days and the systematic changes of the decay rate of the individual outbursts contribute, but they lead to a very similar result for both groups: a very similar value of $\sigma_{\text {res }}$ corresponding to a given $I_{\text {sum }}$ (Fig. 7), irrespective of the time elapsed from the outburst onset. The highest value of $\sigma_{\text {res }}$ at the outburst peak flux (Figs. $4 c$ and $6 b$ ) cannot be accounted for by a variable absorption caused by the accreting matter in the initial phases of the individual outbursts or its gradual decrease in the course of an outburst. The reason is that the $H R$ vs. $I_{\text {sum }}$ diagram (Fig. 11) displays no sign of changes in absorption. We therefore explain the observed variations in $\sigma_{\text {res }}$ as due to the mass inflow rate onto the NS from the disk attaining the highest fluctuations at the outburst peak. These deviations in the mass inflow rate then decrease toward the end of the outburst. It thus takes several days after the onset of outburst for the conditions for irradiation of the disk to stabilize. The decrease in $\sigma_{\text {res }}$ displays a plateau or a bump in group A and at least a change in the decay rate in group B about 10-13 d after the start of outburst. The spiral density waves in a tidally unstable disk become prominent and reach its center, but not earlier than in the second third of the outburst, and manifest themselves as a noise in the X-ray lightcurve, as suggested by the 2D model by Truss et al. (2002). They therefore cannot cause the largest $\sigma_{\text {res }}$ at the outburst peak, but they can be responsible for the behavior in 10-13 d after the onset of outburst. We find evidence of variation in the parameter $C$, expressing the strength of irradiation, during each outburst of group A. According to Dubus et al. (2001), if $C$ remains in a higher, but stable value during outburst, it leads to a decrease in $L_{\max }$ and a considerable 
increase in the outburst duration. However, this is not observed in the Rapid Burster.

Assuming that the mean recurrence time of group A outbursts $T_{\mathrm{C}}=214 \pm 17 \mathrm{~d}$ is due to the stable $\dot{m}_{\mathrm{tr}}$ and stable viscosity of the quiescent disk, we can determine the mean $\dot{m}_{\mathrm{tr}}$ in the following way. When persistent emission dominates, the mass transfer rate at the peak of the outburst, $\dot{m}_{\max }$, can be determined as

$\dot{m}_{\max }=\frac{L_{\mathrm{max}} R_{\mathrm{NS}}}{\zeta G M_{\mathrm{NS}}}$,

where $M_{\mathrm{NS}}$ and $R_{\mathrm{NS}}$ are the mass and radius of the NS, respectively, $\zeta$ refers to the efficiency with which the potential energy is converted to radiation, and $G$ is the gravity constant. Since $\zeta<1$, we find a lower limit of $\dot{m}_{\max }$. We set $M_{\mathrm{NS}}=1.4 M_{\odot}$ and $R_{\mathrm{NS}}=10 \mathrm{~km}$. We obtain $\dot{m}_{\max }>5.1 \times 10^{17} \mathrm{~g} \mathrm{~s}^{-1}$. Integration of the mass accretion rate over the whole mean outburst of group A then yields the total mass accreted onto the NS $m_{\text {accr }}>4.5 \times 10^{23} \mathrm{~g}$. Since the mean $T_{\mathrm{C}}$ is $214 \pm 17$ days, we find that the mean mass transfer rate averaged over the outburst cycle is $\dot{m}_{\mathrm{av}}>4 \times 10^{-10} M_{\odot} \mathrm{yr}^{-1}$. The critical temperature $T_{\text {crit }}$ for the thermal instability depends on the chemical composition of the disk. It is $\sim 6700 \mathrm{~K}$ for an $\mathrm{H}$ disk (e.g. Warner 1995), but can be as high as $\sim 11700 \mathrm{~K}$ for an O disk (Menou et al. 2002). Assuming $R_{\text {disk }}=0.7 R_{\text {lobe }}, P_{\text {orb }}$ of $>1.5 \mathrm{~h}$ and $>0.5 \mathrm{~h}$ are required for the $\mathrm{H}$ and $\mathrm{O}$ disks, respectively, to become thermally unstable. This is consistent with the proposed ultra-compact nature of the Rapid Burster. This value of $\dot{m}_{\text {tr }}$ can also help in resolving the transfer mode. It is close to that in dwarf novae and speaks in favor of a Roche lobe overflow, not of a mass transfer via the wind suggested for GRO J1744-28 by Gosling et al. (2007). We explain the short $T_{\mathrm{C}}$ in the Rapid Burster in terms of the disk irradiation being smaller than in SAX J1808.4-3658 and XTE J1751-305 (as suggested from their differing $I_{\mathrm{T}}$ ), and hence less disk mass consumed in its outburst.

We assess if and how the magnetic field of the NS can influence the properties of the Rapid Burster in quiescence using the $L_{\max }$ vs. $L_{\mathrm{q}} / L_{\max }$ diagram (Fig. 12). Two groups of NS SXTs can be resolved. The fainter and less pronounced one contains two millisecond pulsars with very short $P_{\text {orb }}$ (Ritter \& Kolb 2003) and another ultra-compact candidate, 1H 1905+000 (Jonker et al. 2006). The group A outbursts sort the Rapid Burster to the second, "main group" of NS SXTs, which contains the systems with largely different $P_{\text {orb }}$ including an ultracompact candidate XTE J1709-267 (Jonker et al. 2003). The existence of these two groups can be interpreted as being caused by the different disk viscosity and irradiation of the disk. In quiescence, the mass inflow into the inner disk region is very low and the propeller effect can inhibit accretion (Illarionov \& Siuniaev 1975). Thus, $L_{\mathrm{q}}$ will decrease significantly. Figure 12 shows that the position of the Rapid Burster is consistent with that of other NS SXTs in the "main group", so we argue that any propeller effect is not stronger than in these NS SXTs.

In conclusion, for the Rapid Burster we find very luminous outbursts of a small and light disk (but hardly consumed during outburst), a small vertical dimension of the irradiating source, a relatively high mass transfer rate, an indication of an ultra-short $P_{\text {orb }}$, and no conclusive evidence of the propeller effect.

Acknowledgements. This research made use of the observations provided by the ASM/RXTE team. The support by the grant 205/05/2167 of the Grant Agency of the Czech Republic and the project ESA PRODEX INTEGRAL 90108 and ESA PECS project 98023 is acknowledged. I thank Prof. Harmanec for providing me with the code HEC13. The Fortran source version, compiled version, and brief instructions how to use the program can be obtained via ftp://astro.troja.mff.cuni.cz/hec/HEC13.

\section{References}

Asai, K., Dotani, T., Kunieda, H., et al. 1996, PASJ, 48, L27

Abramowicz, M. A., Czerny, B., Lasota, J. P., et al. 1988, ApJ, 332, 646

Barr, P., White, N. E., Haberl, F., et al. 1987, A\&A, 176, 69

Campana, S., Stella, L., Gastaldello, F., et al. 2002, ApJ, 575, L15

Campana, S., Ravasio, M., Israel, G. L., et al. 2003, ApJ, 594, L39

Campana, S., Ferrari, N., Stella, L., et al. 2005, A\&A, 434, L9

Chakrabarty, D., \& Morgan, H. 1998, Nature, 394, 346

Chen, W., Shrader, C. R., \& Livio, M. 1997, ApJ, 491, 312

Chevalier, C., Ilovaisky, S. A., van Paradijs, J., et al. 1989, A\&A, 210, 114

Dubus, G., Lasota, J.-P., Hameury, J.-M., et al. 1999, MNRAS, 303, 139

Dubus, G., Hameury, J.-M., \& Lasota, J.-P. 2001, A\&A, 373, 251

Falanga, M., Farinelli, R., Goldoni, P., et al. 2004, A\&A, 426, 979

Galloway, D. K., \& Cumming, A. 2006, ApJ, 652, 559

Garcia, M. R., McClintock, J. E., Narayan, R., et al. 1998, in Wild Stars In The Old West, ed. S. Howell, E. Kuulkers and C. Woodward, ASP Conf. Ser., 137, 506

Gierlinski, M., \& Done, Ch. 2002, MNRAS, 337, 1373

Gierlinski, M., \& Poutanen, J. 2005, MNRAS, 359, 1261

Giles, A. B., Swank, J. H., Jahoda, K., et al. 1996, ApJ, 469, L25

Gogus, E., Alpar, M. A., \& Gilfanov, M. 2007, ApJ, 659, 580

Gosling, A. J., Bandyopadhyay, R. M., Miller-Jones, J. C. A., et al. 2007, MNRAS, 380, 1511

Guerriero, R., Fox, D. W., Kommers, J., et al. 1999, MNRAS, 307, 179

Heinzeller, D., \& Duschl, W. J. 2007, MNRAS, 374, 1146

Illarionov, A. F., \& Siuniaev, R. A. 1975, Sov. Astron. Lett., 1, 73

in't Zand, J. J. M., Cornelisse, R., Kuulkers, E., et al. 2001, A\&A, 372, 916

Jonker, P. G., Mendez, M., Nelemans, G., et al. 2003, MNRAS, 341, 823

Jonker, P. G., Bassa, C. G., Nelemans, G., et al. 2006, MNRAS, 368, 1803

Kaluzienski, L. J., Holt, S. S., \& Swank, J. H. 1980, ApJ, 241, 779

King, A. R., \& Ritter, H. 1998, MNRAS, 293, L42

Kouveliotou, C., van Paradijs, J., Fishman, G. J., et al. 1996, Nature, 379, 799

Kunieda, H., Tawara, Y., Hayakawa, S., et al. 1984, PASJ, 36, 215

Levine, A. M., Bradt, H., Cui, W., et al. 1996, ApJ, 469, L33

Lewin, W. H. G., Doty, J., Clark, G. W., et al. 1976, ApJ, 207, L95

Lewin, W. H. G., van Paradijs, J., \& Taam, R. E. 1993, Space Sci. Rev., 62, 223

Lewin, W. H. G., van Paradijs, J., \& Taam, R. E. 1995, in X-ray Binaries (Cambridge Univ. Press), 175

Liller, W. 1977, ApJ, 213, L21

Mahasena, P., Inoue, H., Asai, K., et al. 2003, PASJ, 55, 827

Maitra, D., \& Bailyn, Ch. D. 2004, ApJ, 608, 444

Markwardt, C. B., Swank, J. H., Strohmayer, T. E., et al. 2002, ApJ, 575, L21

Masetti, N. 2002, A\&A, 381, L45

Masetti, N., Frontera, F., Stella, L., et al. 2000, A\&A, 363, 188

Menou, K., Perna, R., \& Hernquist, L. 2002, ApJ, 564, L81

Morrison, R., \& McCammon, D. 1983, ApJ, 270, 119

Nakamura, N., Dotani, T., Inoue, H., et al. 1989, PASJ, 41, 617

Narayan, R., Garcia, M. R., \& McClintock, J. E. 1997, ApJ, 478, L79

Ortolani, S., Bica, E., \& Barbuy, B. 1996, A\&A, 306, 134

Ritter, H., \& Kolb, U. 2003, A\&A, 404, 301

Robinson, E. L., Ivans, I. I., \& Welsh, W. F. 2002, ApJ, 565, 1169

Rutledge, R. E., Lubin, L. M., Lewin, W. H. G., et al. 1995, MNRAS, 277, 523

Rutledge, R. E., Bildsten, L., Brown, E. F., et al. 2001, ApJ, 559, 1054

Shirey, R. E., Bradt, H. V., Levine, A. M., et al. 1996, ApJ, 469, L21

Spruit, H. C., \& Taam, R. E. 1993, ApJ, 402, 593

Šimon, V. 2000, A\&A, 354, 103

Šimon, V. 2002, A\&A, 381, 151

Šimon, V. 2004, A\&A, 418, 617

Šimon, V. 2006, in Populations of High Energy Sources in Galaxies, Proc. of the 230th IAU Symp., ed. E. J. A. Meurs and G. Fabbiano (Cambridge University Press), 47

Truss, M., \& Done, Ch. 2006, MNRAS, 368, L25

Truss, M. R., Wynn, G. A., Murray, J. R., et al. 2002, MNRAS, 337, 1329

van Paradijs J. 1996, ApJ, 464, L139

Vondrák, J. 1969, Bull. Astron. Inst. Czechosl., 20, 349

Vondrák, J. 1977, Bull. Astron. Inst. Czechosl., 28, 84

Wachter, S., Hoard, D. W., Bailyn, Ch.D., et al. 2002, ApJ, 568, 901

Warner, B. 1995, Cataclysmic Variable Stars, (Cambridge: Cambridge Univ. Press)

Wijnands, R. 2006, Trends in Pulsar Research, by J. A. Lowry (New York: Nova Science Publishers, Inc., New , NY USA), 53

Wijnands, R., \& Wang, Q. D. 2002, ApJ, 568, L93 